\title{
Maintaining Efficiency While Integrating Entrants From Lower-Performing Environments: An Experimental Study*
}

\author{
Timothy C. Salmon ${ }^{\dagger}$ \\ Florida State University \\ Roberto Weber \\ Carnegie Mellon University
}

August 2009

\begin{abstract}
Efficient growth often requires the integration of individuals from lower-performing groups, firms, or societies into higher-performing ones. Such integration may be difficult without facilitating interventions or restrictions. We explore, using a laboratory experiment, the effectiveness of two regularly-employed entry restrictions: entry quotas and entry exams. We use a coordination game with Pareto-ranked equilibria, in which we allow an efficiently-coordinated group and an inefficiently-coordinated one to arise endogenously. We then allow individuals to move from the low-performing group to the better one. We vary whether such movement is unrestricted, is limited to one entrant per period, or is subject to passing an entry exam. We find both kinds of restrictions improve the efficient integration of entrants, but that there is no additional benefit obtained by their combination. The restrictions lead both to improved behavior among entrants and to the maintenance of good behavior among incumbents in the high-performing group.
\end{abstract}

JEL Codes: C72, C92, M12

Key Words: Growth, Entry, Coordination, Experiments

\section{Introduction}

An important issue in the pursuit of large efficiently-performing groups is how to incorporate new entrants. For example, a growing economy might need to rely on immigration to provide a suitable labor supply. Similarly, profitable firms often expand by integrating individuals through external hiring.

In most cases, the growing group is one that has previously performed well on some important dimension, such as productivity or profitability, leading to a desire to maintain such high performance while undergoing growth. Incumbents in the growing entity have a history of working well together, expecting high performance from one another, and

${ }^{*}$ The authors would like to thank the National Science Foundation for research support in funding these experiments.

${ }^{\dagger}$ Florida State University, Department of Economics, 113 Collegiate Loop, Room 263, PO Box 3062180, Tallahassee, FL 32306-2180, tsalmon@fsu.edu. Phone: 850-644-7207 Fax: 850-644-4535.

${ }^{\ddagger}$ Deparment of Social \& Decision Sciences, Carnegie Mellon University, Pittsburgh PA 15213, robertoweber@cmu.edu. Phone: 412-268-3224 Fax: 412-268-6938. 
obtaining efficient coordination of their actions. Conversely, entrants are often from lowerperforming entities, such as poorer nations or less profitable firms, where expectations of others' behavior are lower and where the resulting behavior is either coordinated on less efficient equilibria, or perhaps not coordinated at all. This creates an important concern: when incorporating entrants from low-peforming groups, how can growing entities maintain the high performance that has made them successful? This is particularly important in contexts with a high degree of action interdependence, where small numbers of deviations from the high behavioral standards previously obtained by the growing entity can lead to low performance. In order to grow successfully while incorporating new entrants, it must be that both new entrants adapt their behavior to the higher standards of the better-performing group and also that incumbents in the high-performing group maintain the good behavior that has previously led to success.

In this paper, we explore two common policies for facilitating the successful entry of individuals from lower-performing groups into ones that have historically performed more efficiently. The first policy we examine is an entry quota, whereby the number of entrants in a given time period is regulated. For example, a country may limit the number of immigrants that are allowed entry in a given year, as the United States did with the 1924 Immigration Act. Similarly, a club may limit the number of new members it accepts. Or a firm may explicitly target a slow-growth strategy as a way to maintain efficient practices. The second policy is an entry exam, whereby individuals are required to demonstrate knowledge or skills prior to being granted entry. For example, countries set thresholds for granting entry to immigrants, such as sufficiently high professional or educational standing, or may implement naturalization exams as a requirement for citizenship. A club may require entrants to know about the club's history prior to entry. Finally, many firms use pre-employment testing as a way of screening potential job applicants for desirable qualities.

We focus on a production context in which there is high action interdependence - using a "minimum-effort" or "weak-link" coordination game, in which efficiency (output) is determined by the lowest input by any group member. Thus, in order to perform well, a group needs all players to make high input choices, making it particularly difficult to incorporate new entrants since efficiency can be harmed if either any entrant or any incumbent selects lower input. Moreover, growth in such environments may be particularly difficult because larger group sizes have a harder time obtaining efficient coordination (see Van Huyck et al., 1990; Weber 2006).

In a standard game of this sort there is some number $N$ of players who each select from an ordered set of strategies $x_{i} \in(1, \ldots, K)$ and produce some collective output that is an increasing function of the minimum choice among all the players. Players benefit from higher output levels, but selecting higher strategies is costly - players only want to select a higher strategy if all other players do so as well. The set of pure-strategy Nash equilibria for the game consists of all outcomes in which players select the same strategy. The incentives are such that equilibria corresponding to higher effort choices are more efficient than those in which players make lower choices and the efficient equilibrium corresponds to all players selecting strategy $K$. Our version of the game is augmented by including an element which leads to the group output being an increasing, concave function of the number of players in the group. Thus, holding the minimum choice in the group constant, output is greater when the group is larger. This creates a ceteris paribus efficiency gain from having larger 
groups. It is still the case, though, that growth in the size of the group decreases earnings if it leads to coordination on a lower-output equilibrium.

In our experiment, we divide each group into two endogenously-determined subgroups - one "high-performing" small subgroup and one "low-performing" large subgroup. Our procedure reliably creates small, efficiently-coordinated subgroups and large subgroups coordinated on inefficient levels of output. Earnings in the small groups are higher.

We then allow participants from the Low subgroup to voluntarily move to the High subgroup. Given our payoff structure, such movement can lead to a Pareto improvement whereby both incumbents in the High subgroup and entrants from the Low subgroup can earn higher payoffs. But this improvement is only realized if entry does not lead to lower output levels.

We consider four treatments, corresponding to varying degrees of entry regulation. In a Baseline treatment, we impose no restrictions, allowing anyone who wants to move to do so. In a Quota treatment, only one participant is allowed to move per period, with multiple requests accepted or declined by a random lottery. In a Quiz treatment, there is no limit on the number of entrants, but all entrants must successfully complete a short quiz demonstrating both the ability to calculate game payoffs and knowledge of historical outcomes in the High subgroup. Finally, in a Combined treatment, both restrictions are simultaneously in place.

Our goal is to explore the extent to which the above two restrictions are effective at obtaining successful entry by individuals from a low-performing group into a higher-performing one. We include the combined treatment to determine the extent to which there are benefits from employing multiple policies simultaneously.

Previous research in economics, organizations, and sociology explores the determinants of successful assimilation of new entrants into entities such as countries, firms, or work teams (Borjas, 1995; Cable and Judge, 1996; Card, 2005; Chatman and Flynn, 2001; Friedberg, 2001; Ortega, 2005; Rumbaut, 1997). Some of this work explicitly addresses the need for and effectiveness of different policies to regulate entry. For example, Warne (1921) argued for the importance of government policies to regulate and ensure the assimilation of immigrants into U.S. society, noting that, "If the volume of total immigration is likely at any time to become so large as to make ineffective our forces of assimilation, then this volume should be decreased by restrictive measures; if the elements comprising any particular group or race, no matter how few in number relatively, are unassimilable into our American life then these too should be restricted" (p. 185). More recently, Borjas (1994) discussed how changes to a points-based scoring system used to evaluate immigrants to Canada significantly affected the educational attainment of admitted immigrants. In an organizational context, Ryan and Sackett (1987) demonstrated that pre-employment screening can be useful for discriminating between honest and dishonest employees. O' Reilly III, Chatman, and Caldwell (1991) demonstrated that greater correspondence between an individual's characteristics and the organization's culture yields more successful outcomes in hiring, such as longer employee retention. Our work adds to this literature, by employing a laboratory experiment to explore different policies for regulating entry. While the laboratory necessarily sacrifices some degree of generalizability to these real-world contexts, it also allows us to avoid concerns that are present in the field. For example, in evaluating the effectiveness of different policies for regulating entry in real-world groups, the endogenous selection of policies makes inference 
about whether outcomes are truly the result of a particular policy difficult. In the laboratory, however, we can avoid this concern by randomly-assigning groups to different policies.

Our work is more closely related to a small number of experimental studies that explore alternate methods for determining group composition, and their effect on efficiency in production games. For example, Weber (2006) shows how groups that start of small and grow can maintain efficient coordination, but only when entrants are exposed to a group's prior history. However, entry in Weber's experiment drew from participants with no prior history of playing the game. In our case, entry involves incorporating participants with a history of low-efficiency outcomes. This difference in the prior experience of entrants is important since in many real contexts growth involves incorporating entrants from lower-performing groups. The past poor performance of these entrants' groups may make their efficient assimilation more difficult than when they have no history. In a public goods framework, Ahn, Isaac and Salmon $(2008,2009)$ explore how endogenous group selection influences contributions, with players having the opportunity to enter and exit groups. Their experiments vary the degree to which both entry and exit from groups are restricted, finding that both restricted entry and exit significantly influence contributions.

\section{Experimental Design}

In our experiment subjects played a version of a weak-link coordination game over a total of 25 periods. Earnings from the game were denominated in Experimental Currency Units (ECU), which were converted to money at a rate of $60 \mathrm{ECUs}=\$ 1$.

\subsection{The Game}

In each period subjects had 7 tokens that they could either keep or contribute to a group account. We will let $x_{i}$ denote the amount contributed. For every token retained the subject received 3 ECUs. The total return on the tokens contributed to the group account depended on the number of players in the group, $N$, and the minimum of the tokens contributed among the group $(N)$. Let $x_{-i}$ represent the vector of contributions of all of the other members of $i$ 's group. Then the total return on the group contribution was $6 * N^{.25} * \min \left(x_{i}, x_{-i}\right)$. We will refer to $6 * N^{.25}$ as the group account multiplier since it tells us the per token return for the minimum number of tokens contributed to the group account. Finally, contributing to the group account cost an individual $x_{i}^{1.5}$. The entire payoff function is contained in equation 1

$$
\pi\left(x_{i}, x_{-i}\right)=3 *\left(7-x_{i}\right)+6 * N^{.25} * \min \left(x_{i}, x_{-i}\right)-x_{i}^{1.5}
$$

The properties of this game are standard for weak-link games as described above. For any group size $N \geq 2$, any level of contribution is a Nash equilibrium if all players are contributing the same amount. All players contributing 7, or the maximum number of tokens, is the Pareto dominant or efficient equilibrium. To make certain that subjects would be able to understand the incentives generated by this payoff function we provided them with multiple payoff tables corresponding to differing values of $N$ and we provided the ability in the software to calculate prospective earnings by entering a hypothetical minimum 
for others as well as an own contribution to see the resulting payoff for as many combinations as they liked prior to making a decision in a period.

\subsection{Stages}

Each session involved 20 subjects who were split into 2 separate 10-person groups. These groups were fixed for the duration of the session and there was never any interaction across those groups. The experiment proceeded in three stages.

In Stage 1 which consisted of periods 1-3, all members of a 10-person group played the weak-link game together. In the instructions for this stage, subjects were given a payoff table corresponding to $N=10$ to which they could refer in making their choices. At the end of every period in Stage 1, subjects saw their own contribution, the minimum contribution in the group, the group account multiplier for the group, and their own earnings for the period.

Stage 2 took place over periods 4-10. At the beginning of period 4, each 10-person group was split into two sub-groups which we called the "High" and "Low" sub-groups. The High sub-group consisted of the two members from the 10-person group who had contributed the most in Stage 1. The Low sub-group consisted of the other 8 who were obviously the lowest 8 contributors during Stage 1. Subjects were not told prior to this event that they would be divided in this way so their Stage 1 contributions could not have been made strategically to try to get into the High (or Low) sub-group. Subjects received additional verbal and written instructions about the new subgroups, including additional payoff tables corresponding to values of $N=2$ and $N=8 .^{1}$ The experiment software told subjects to which group they were assigned and gave them a complete contribution history of their fellow group members. The instructions used the labels "High" and "Low" to refer to the sub-groups, and were also explicit about how the subgroups were determined. Subjects then began Stage 2 of the experiment, playing the weak-link game with only those in their 2 or 8-person subgroup. After every period in Stage 2 subjects saw the same information as before about their own subgroup but they were also given information about what was happening in the other subgroup including the number of group members, the group account multiplier and minimum group contribution.

Our expectation was that the High sub-groups would be able to coordinate on a high level of contributions by the end of Stage 2, while the Low sub-groups would fail to achieve high coordination and would instead play several periods with low minimum group contributions. This was necessary in order to set up Stage 3 of the experiment in which we allowed subjects in the Low sub-group the opportunity to move to the High sub-group. Stage 3 lasted from period 11 through the final period, 25. Prior to each period, subjects still in the Low subgroup were asked if they wished to move to the High sub-group. Moves into the High subgroup were irreversible and subjects who began in the High sub-group were never allowed the option of moving into the Low sub-group. We chose this limited flexibility to allow us to precisely address our main research question of whether successful groups could maintain their level of efficient coordination during an influx from the lower performing group. Other than the ability to transition groups, this stage proceeded the same as in Stage 2 in terms of the information provided to the subjects at the end of a period.

\footnotetext{
${ }^{1}$ Copies of all instruction scripts are available from authors.
} 


\subsection{Treatments}

The mechanism underlying the group transitions varied according to our four treatments. Complete verbal and written instructions were provided to members of both sub-groups about the group transition mechanism that would be used. This is important, as it meant that members of the High sub-group knew what restrictions governed entry into their subgroup. Also, at the beginning of each period, following any movement from the Low subgroup to the High subgroup, all subjects saw the number of people in each of the two subgroups and had the opportunity to explore payoffs for their current (and possibly new) group size.

In the first, Baseline, treatment any subject indicating a desire to move from the Low sub-group into the High one was allowed to do so. At the beginning of a period, Low subgroup subjects wishing to move indicating this choice on the computer and were then moved into the High subgroup. Thus, there were no restrictions on movement from the Low to High sub-group. The other three treatments involved some degree of restrictions.

In our second, Quota, treatment only one subject was allowed to move from the Low to High sub-group per period. This was implemented by the software randomly selecting one subject from among all those expressing a desire to change sub-groups to actually do so, while the remaining subjects wishing to move were forced to remain in the Low subgroup. As in the Baseline, at the beginning of a period subjects in the Low subgroup again indicated whether they wished to move to the other subgroup. On the next screen, they found out whether they had been able to do so.

In the third, Quiz, treatment subjects had to correctly answer a two question quiz in order to move between sub-groups. Any subjects expressing an interest in moving and successfully answering the quiz questions were allowed to move to the High subgroup. One question asked the subject something about the history of the High group from the prior period (such as the size of the group or the minimum contribution level). The other question gave them a sample set of contributions and asked them to calculate the payoff for one of the players. We gave them extensive instructions on how to do this calculation and the software pre-calculated for them the group account multiplier, $6 * N^{.25}$, as well as the cost of contributing, $x_{i}^{1.5}$, which made the calculation quite straightforward. We also provided subjects with access to the standard Windows calculator.

The last treatment is the Combined mechanism, as it imposes both a quiz and a quota restriction. In this treatment all subjects wishing to transition answered the same two kinds of questions as in the Quiz treatment. But among all those getting both answers correct, only one was allowed to move.

We conducted 9 sessions of this experiment for a total of 18 groups and 180 subjects. Eight of the sessions were conducted at the xs/fs lab at Florida State University with 2 sessions of each treatment or 4 independent 10-person groups per treatment. We also have one additional session of the Combined treatment which was conducted at the Pittsburgh Experimental Economics Laboratory (PEEL) at the University of Pittsburgh. Average total earnings were $\$ 22.03$ (including a $\$ 10$ participation fee) for an experiment lasting 1 to 1.5 hours. The software used for this experiment was programmed in Z-Tree, Fischbacher (2007). 


\begin{tabular}{ll|cc|cc|cc|cc}
\hline \hline & & \multicolumn{2}{|c|}{ Contribs } & \multicolumn{2}{c|}{ Group Min } & \multicolumn{2}{c|}{ Group Size } & \multicolumn{2}{c}{ Profits } \\
& & $4-10$ & $16-25$ & $4-10$ & $16-25$ & $4-10$ & $16-25$ & $4-10$ & $16-25$ \\
\hline \hline Low & Baseline & 1.8 & 2.2 & 1.0 & 2.4 & 8 & 2.2 & 22.7 & 25.7 \\
Groups & Quota & 1.1 & 3.1 & 0.5 & 2.9 & 8 & 1.6 & 21.2 & 26.2 \\
& Quiz & 1.2 & 1.1 & 0.6 & 0.9 & 8 & 2.2 & 22.1 & 23.8 \\
& Combined & 1.4 & 0.8 & 1.0 & 0.5 & 8 & 2.6 & 24.8 & 21.8 \\
\hline \hline High & Baseline & 4.0 & 0.5 & 3.6 & 0.3 & 2 & 8.1 & 25.1 & 21.7 \\
Groups & Quota & 4.5 & 3.0 & 4.0 & 2.7 & 2 & 9.2 & 25.8 & 35.9 \\
& Quiz & 5.5 & 3.8 & 5.0 & 3.4 & 2 & 8.5 & 26.8 & 35.4 \\
& Combined & 4.9 & 3.4 & 4.6 & 3.3 & 2 & 7.6 & 27.5 & 37.2 \\
\hline \hline
\end{tabular}

Table 1: Summary statistics from Low and High groups by period and treatment.

\section{Results}

Recall that in our experiment, each 10-person group remained fixed for the entire experiment. However, in Stages 2 and 3, the 10-person group consisted of a High and a Low subgroup of potentially varying size. For simplicity, in the rest of the paper we refer to these subgroups using the term "group," and when we use the terms "group minimum" or "group size" we are referring to the subgroups. We explicitly note any instances in which we are referring to the entire 10-person group.

\subsection{Summary Statistics}

We begin by first presenting a series of summary statistics and graphical depictions of the data, before presenting the formal results through a series of regressions. Table 1 presents a first look at the data, summarizing two individual-level variables (average overall contributions and average overall profits) and two group-level variables (average group size and average group minimum) for each treatment. The results, are presented separately for Stage 2 (periods 4-10) and for the last 10 periods from Stage 3 (16-25). The reason to present the summary statistics from those ranges is that the Stage 2 numbers demonstrate how well the two kinds of groups perform prior to the experimental treatment, and thus whether our Stage 2 manipulation did indeed produce differentially-efficient "low" and "high" groups, while the last 10 periods of Stage 3 demonstrate the overall effect of the treatment.

There are a number of features that are readily apparent in the summarized data. First, we see that indeed the Low groups fail to coordinate on a good equilibrium in Stage 2 (the average contribution level is generally around 1) while the High groups are able to coordinate on more efficient equilibria (the average contribution levels are between 4 and 5 ). Thus, our Stage 2 group assignment worked as we expected. Second, in Stage 3, most of the Low group subjects choose to move into the High group, though in every treatment there are some occasions in which subjects chose to stay in the Low group.

Next, if we look at average contributions and minima in Stage 3 for the High groups we see that in the Baseline contributions collapse to near 0 on average while in the three treatments with entry restrictions the High groups are generally able to maintain coordination on more efficient (higher) equilibria, though with perhaps some backsliding relative to their 
average contributions in Stage 2. The Low group contributions are not our main focus, but we interestingly find that in some of the Low groups the few remaining subjects by periods 16-25 are sometimes able to improve the efficiency of coordination (reflected both in higher average contributions and group minima, relative to Stage 2). Since our primary interest is in what happens in the High groups, and particularly in how entry into the High groups responds to our treatments, we largely ignore contributions and minima in the Low groups in the remainder of our analysis. Finally we see that subjects' profits generally follow the pattern indicated by the contributions and minima. That is, profits are better for High than Low group subjects (except in the Baseline) and profits for High group subjects are higher in the three treatment conditions than in the Baseline.

Interestingly, we find little difference between the High groups in the three treatments for periods 16-25 in contributions, minima or earnings. This suggests that the Quota and Quiz mechanisms generally work equally well, and that there is no substantive gain from having both mechanisms in place. We explore these differences more precisely in the next section.

Figures 1 and 2 examine the time paths of these results, showing the averages of each of the summary statistics for each period in the High groups and by treatment. ${ }^{2}$ The trends displayed in the figures display the same characteristics indicated by the summary statistics. In the three treatments with restrictions on entry average contributions and minima drop modestly from Stage 2 to Stage 3, while in the Baseline they start at about the same level but then collapses to almost zero. The earnings graph in Figure 2 similarly shows the earnings per period in the three treatments to be well above the earnings per period in the Baseline

Looking at entry into the High group ("Group Size" in Figure 2), we see a picture of the time pattern of how group size evolves. While the Baseline and Quiz result in nearly identical total group movement in the first five periods of Stage 3, the Baseline had a larger growth spurt in the first period or two with the growth in the Quiz treatment catching up soon after. The Quota and Combined treatments not surprisingly lead to slightly slower growth but we observe convergence to similarly large group sizes in later periods across all treatments.

Figure 3 shows more detail on group movement. The graph presents the average number of attempted and successful movements between groups, by treatment, for periods in which at least one move was attempted. This figure shows that, in Period 11, there are approximately the same number of attempted moves in all three treatments as in the Baseline. In the three treatments with restrictions, many of those initial attempts fail in Period 11 leading there to be substantially fewer successful moves in those treatments than in the Baseline. After Period 11, while there are more attempted moves in the three treatments than in the Baseline, there are approximately the same number of successful moves in all treatments. Therefore, while the treatments limiting movement produce a large difference in the number of successful moves in the first period in which movement is allowed, their effect on total movement in subsequent rounds is not substantial.

There is, however, a potentially important difference in movement between the Quiz treatment and the Quota and Combined treatments. In the latter two, the average number

\footnotetext{
${ }^{2}$ We do not provide similar figures for the Low groups to conserve space and again due to the fact that their behavior following the transition to Stage 3 is not the element of interest.
} 


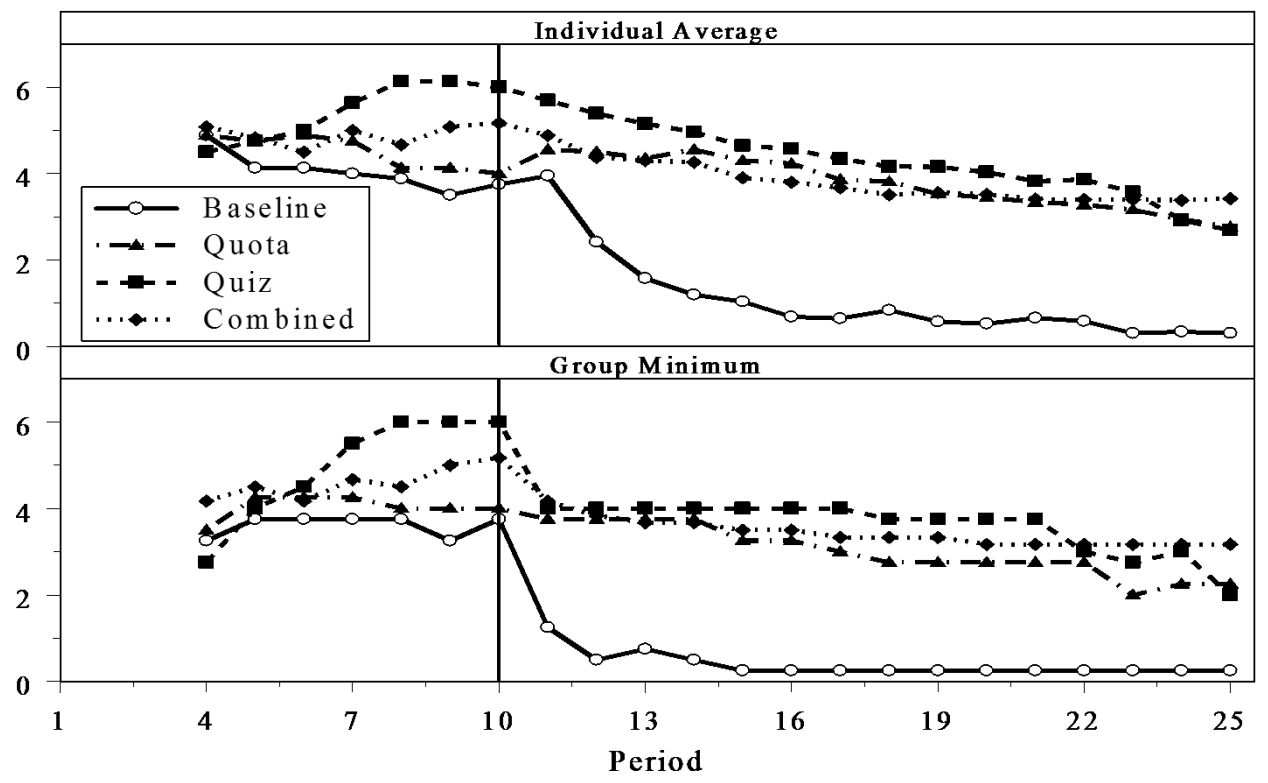

Figure 1: Averages of individual and group minimum contributions for High groups.

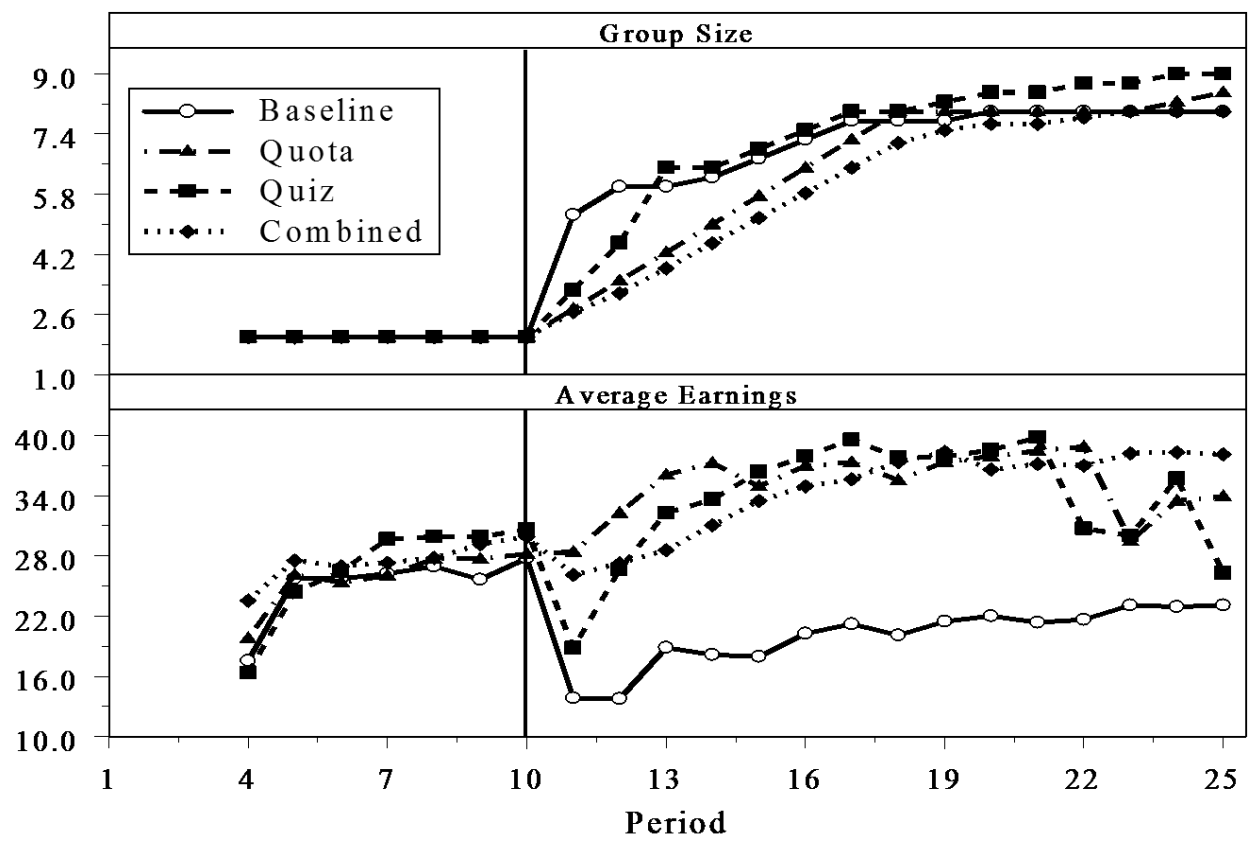

Figure 2: Average group size and individual earnings for High groups. 


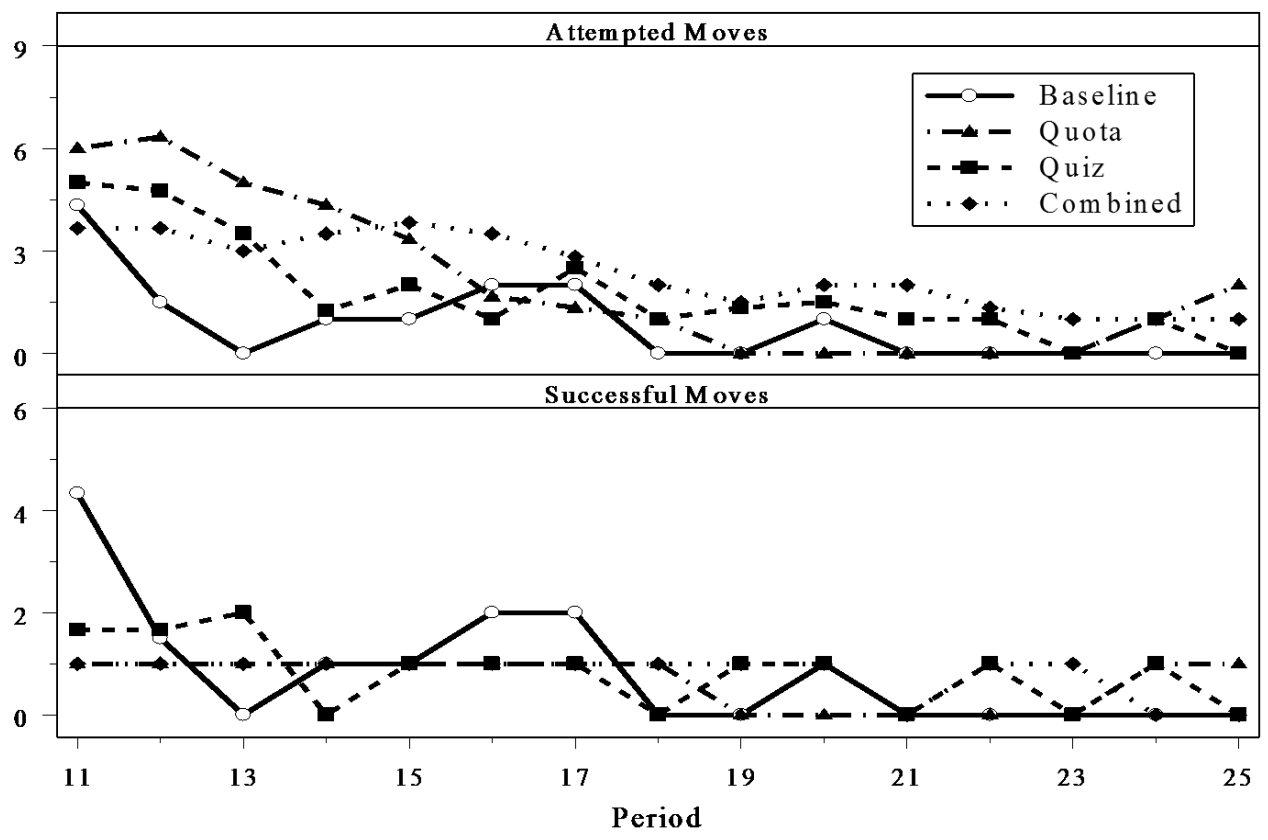

Figure 3: Average attempted and successful moves per period per group with attempted moves in that period.

of successful moves per period is 1 but that is also the maximum and minimum number of moves as well due to the enforced quota mechanism. ${ }^{3}$ In the Quiz treatment, however, we see that the average number of successful moves per group is around 2 for periods 11-13 and since there was no explicit limit on the number of moves allowed in a period there is also variation in this number, with some groups experiencing perhaps 3-4 successful moves and other experiencing only 1 . This heterogeneity in successful moves could well lead to differential behavioral consequences in the Quiz treatment, relative to the Quota and Combined treatments, as the High groups in the Quiz treatment potentially have to incorporate a steady influx of newcomers at a higher rate in those early periods than High groups in either the Quota or Combined treatment. But as Figures 1 and 2 reveal, the differential effects on outcomes of such faster entry are minimal.

For our first set of basic statistical results we provide a set of non-parametric Wilcoxon rank-sum tests on whether group-level averages differ between the treatments and the Baseline in each phase. The $p$-values resulting from these pairwise tests are in Table 2. For each test, an observation consists of the average contribution (or average group minimum) over a stage for each group meaning we have four total observations for the Baseline, Quiz and Quota treatments and six observations for the Combined treatment. Given that we are aggregating across all periods in a stage for each group, this test represents a very conservative test of whether or not there were differences across treatments. Examining the results of the tests in Table 2 we see that there are no differences between either average

\footnotetext{
${ }^{3}$ There are instances of groups with 0 moves per period, but the data for this figure excludes those as the interest is on examining the attempt/success rate of moves and not the attempt rate of moves.
} 


\begin{tabular}{lcccc}
\hline \hline & \multicolumn{2}{c}{ Average of Individual Contributions } & \multicolumn{2}{c}{ Minimum of Group Contributions } \\
& Periods 4-10 & Periods 16-25 & Periods 4-10 & Periods 16-25 \\
\hline \hline Quota & 0.886 & 0.057 & 0.886 & 0.037 \\
Quiz & 0.686 & 0.029 & 0.468 & 0.027 \\
Combined & 0.831 & 0.010 & 0.669 & 0.013 \\
\hline
\end{tabular}

Table 2: P-Values from Wilcoxon Rank-Sum tests of differences in distributions of High group averages between treatments and baseline.

contributions or group minimum contributions in Stage 2, i.e. periods 4-10. On the other hand, in the last 10 periods of Stage 3, periods 16-25, we find that all of the treatments are significantly different from the Baseline with regard to both overall average contributions and group minimum. While we have not included the results from the tests, none of the three treatments are found to be significantly different from each other.

\subsection{Regression Analysis}

For a more detailed regression analysis of treatment effects, we employ an estimation strategy that allows us to examine the treatment effects in Stage 3 conditional on behavior in Stage 2. We employ a standard differences-in-differences structure for panel data, in which we specify binary variables for the different treatment effects activated throughout the sessions, Quota, Quiz and Combin, a binary variable corresponding to Stage 3, S3, and then interactions of those treatment effects with the Stage 3 dummy. These interaction terms measure any difference between behavior after the introduction of the group transition mechanism treatment has occurred (in Stage 3) but conditioned on the behavior in that population prior to the treatment being implemented. For the full specification see equation 2. In order to accommodate any subject- or group-level heterogeneity we use a random effects panel structure with the random effect, $c_{i}$, on the individual or group level depending on the regression. The regressions at the individual level include robust standard errors clustered at the subject level but due to the low number of clusters for the group level regression we will only use robust standard errors. Since our regressions will be conducted separately for those in High groups and Low groups we want to begin this analysis after those group identities have been established. Consequently these regressions do not include data prior to period 4 .

$$
\begin{gathered}
y_{i t}=\alpha_{0}+\alpha_{1} G S_{i}+\alpha_{2} \text { Period }+\alpha_{3} \text { Quota }+\alpha_{4} \text { Quiz }+\alpha_{5} \text { Comb }+ \\
\beta_{0}(L G * S 3)+\beta_{3}(L G * S 3 * \text { Quota })+\beta_{4}(L G * S 3 * \text { Quiz })+\beta_{5}(L G * S 3 * \text { Comb })+ \\
\gamma_{0}(H G * S 3)+\gamma_{1}\left(H G * S 3 * G S_{i}\right)+\gamma_{2}(H G * S 3 * \text { Period })+ \\
\gamma_{3}(H G * S 3 * \text { Quota })+\gamma_{4}(H G * S 3 * \text { Quiz })+\gamma_{5}(H G * S 3 * \text { Comb })+c_{i}+\varepsilon_{i t}
\end{gathered}
$$

Our first regressions look at individual contributions and earnings using data from each individual per period as the unit of observation. To allow us to cleanly separate the behavioral treatment effects among those subjects who were initially in the High group from 
those who were initially in the Low group we run separate regressions for both types of subjects. For the subjects initially in the Low groups they have two sets of Stage 3 treatment variables. One set measures the effect of being in a particular treatment on behavior while still in the Low group and the other set measures the behavior while in the High group (if a move has occurred). Since those initially in the High group are restricted to be only in that group, they have only one set of Stage 3 interactions. These regressions can be found in the first four columns of Table 3.

The key variables for this study are the Stage 3 treatment variables for subjects in the High groups, $\gamma_{3}, \gamma_{4}$ and $\gamma_{5}$. The fundamental question motivating this study is the degree to which any of the treatment mechanisms facilitates efficient coordination (higher contributions) in the High group once entry from the Low group is allowed in Stage 3. Thus, we are interested in how the treatment mechanisms influence both whether subjects originally in the Low group make higher contributions once in the High group in Stage 3 and whether subjects originally in the High group make high contributions in Stage 3. The regressions show results consistent with the summary statistics from the prior section. By examining the regressions on individual contributions we see that, for those initially in the Low group who move to the High group in Stage 3, conditional on being in one of the three treatments their contributions are on average 3.6 units higher than for subjects who move in the Baseline condition as indicated by the values of $\gamma_{3}, \gamma_{4}$ and $\gamma_{5}$. in the first column. For those initially in the High group, contributions drop by about 1.6 in the Baseline treatment in Stage 3, $\gamma_{0}$ in the second column, while contributions in the other three treatments are about 2 units above that, $\gamma_{3}, \gamma_{4}$ and $\gamma_{5}$. The overall effect of the treatments on contributions in Stage 3 for those initially in the High group can be calculated by taking a linear combination of the Phase 3 binary variable (which measures the omitted treatment, Baseline) with the relevant interaction, $\gamma_{0}+\gamma_{3,4}$, or 5 . These come out to be $0.267,0.566$ and 0.338 for the Quota, Quiz and Combined treatments respectively with none of those total effects being significantly different from 0 . Thus, on net, subjects who begin in the High groups do not drop their contributions once entry takes place in Stage 3, while they do drop their contributions in the Baseline condition.

The regressions in the third and fourth columns of Table 3 show similar results for subjects' earnings. By examining the same coefficients again we see that earnings for those that began in the Low Group increase substantially when they move to the High group in the three treatments but not in the Baseline, where they actually decline. For those that begin in the High groups, their earnings fall substantially in the Baseline treatment during Stage 3 but again by testing linear combinations of the Baseline effect with the interactions we see that earnings do not fall during Stage 3 in the three treatments.

Further evidence regarding the effectiveness of the treatments can be found in the last column of Table 3, which presents a similar regression using the minimum contribution in a group in a period as the unit of observation. We only conduct this regression for the High groups, since this is our focus. We see that the group minimum drops significantly during Stage 3 in the Baseline condition, $\gamma_{0}$ in the last column, while minima in the three treatments are significantly above the Baseline, $\gamma_{3,4}$ and 5 . Tests on the linear combinations to identify the total effect on the group minima for the treatments again show that there is no statistically significant difference between Stage 2 and Stage 3 for the three treatments.

Another observation from Table 3 is that there again appears to be no difference between 


\begin{tabular}{|c|c|c|c|c|c|}
\hline & \multicolumn{2}{|c|}{ Individual Contrib } & \multicolumn{2}{|c|}{ Individual Earnings } & \multirow{2}{*}{$\begin{array}{c}\text { Group Min } \\
\text { High }\end{array}$} \\
\hline & Initially in Low & Initially in High & Init in Low & Init in High & \\
\hline GroupSize, $\alpha_{1}$ & $\begin{array}{c}-0.092^{* *} \\
(0.045)\end{array}$ & $\begin{array}{l}-0.045 \\
(0.056)\end{array}$ & $\begin{array}{c}0.196 \\
(0.185)\end{array}$ & $\begin{array}{c}0.714^{* * *} \\
(0.269)\end{array}$ & $\begin{array}{c}-0.190^{* * *} \\
(0.043)\end{array}$ \\
\hline Period, $\alpha_{2}$ & $\begin{array}{c}-0.0384^{* *} \\
(0.019)\end{array}$ & $\begin{array}{c}-0.113^{* * *} \\
(0.020)\end{array}$ & $\begin{array}{c}0.269^{* * *} \\
(0.053)\end{array}$ & $\begin{array}{c}0.309^{* * *} \\
(0.106)\end{array}$ & $\begin{array}{c}>-0.001 \\
(0.021)\end{array}$ \\
\hline Quota, $\alpha_{3}$ & $\begin{array}{c}-0.647^{* * *} \\
(0.219)\end{array}$ & $\begin{array}{c}0.464 \\
(1.060)\end{array}$ & $\begin{array}{l}-0.643 \\
(1.247)\end{array}$ & $\begin{array}{l}0.747 \\
(2.529)\end{array}$ & $\begin{array}{l}0.429 \\
(1.160)\end{array}$ \\
\hline Quiz, $\alpha_{4}$ & $\begin{array}{c}-0.710^{* * *} \\
(0.232)\end{array}$ & $\begin{array}{l}1.411 \\
(0.970)\end{array}$ & $\begin{array}{l}-1.525 \\
(1.323)\end{array}$ & $\begin{array}{l}1.654 \\
(2.347)\end{array}$ & $\begin{array}{l}1.357 \\
(1.359)\end{array}$ \\
\hline Combined, $\alpha_{5}$ & $\begin{array}{c}-0.400^{*} \\
(0.237)\end{array}$ & $\begin{array}{l}0.869 \\
(1.016)\end{array}$ & $\begin{array}{l}2.071 \\
(1.334)\end{array}$ & $\begin{array}{l}2.365 \\
(2.118)\end{array}$ & $\begin{array}{l}0.988 \\
(1.107)\end{array}$ \\
\hline Low Group $*$ Stage $3, \beta_{0}$ & $\begin{array}{l}0.296 \\
(0.376)\end{array}$ & & $\begin{array}{l}0.307 \\
(1.503)\end{array}$ & & \\
\hline LGS3 $*$ Quota, $\beta_{3}$ & $\begin{array}{l}-0.165 \\
(0.407)\end{array}$ & & $\begin{array}{l}-1.521 \\
(1.577)\end{array}$ & & \\
\hline LGS3 $*$ Quiz, $\beta_{4}$ & $\begin{array}{l}0.270 \\
(0.468)\end{array}$ & & $\begin{array}{c}-2.910^{*} \\
(1.575)\end{array}$ & & \\
\hline LGS3 $*$ Combined, $\beta_{5}$ & $\begin{array}{l}-0.505 \\
(0.414)\end{array}$ & & $\begin{array}{c}-2.621^{*} \\
(1.563)\end{array}$ & & \\
\hline High Group $*$ Stage $3, \gamma_{0}$ & $\begin{array}{l}0.405 \\
(0.537)\end{array}$ & $\begin{array}{c}-1.642^{*} \\
(0.953)\end{array}$ & $\begin{array}{c}-4.942^{* *} \\
(2.304)\end{array}$ & $\begin{array}{c}-11.80^{* * *} \\
(2.982)\end{array}$ & $\begin{array}{c}-2.222^{* * *} \\
(0.451)\end{array}$ \\
\hline HGS3 $*$ GroupSize, $\gamma_{1}$ & $\begin{array}{c}0.177^{* *} \\
(0.071)\end{array}$ & & $\begin{array}{c}0.854^{* * *} \\
(0.324)\end{array}$ & & \\
\hline HGS3 ${ }^{*}$ Period, $\gamma_{2}$ & $\begin{array}{c}-0.133^{* * *} \\
(0.024)\end{array}$ & & $\begin{array}{c}-0.398^{* * *} \\
(0.133)\end{array}$ & & \\
\hline HGS3 $*$ Quota, $\gamma_{3}$ & $\begin{array}{c}3.690^{* * *} \\
(0.415)\end{array}$ & $\begin{array}{l}1.909^{*} \\
(1.023)\end{array}$ & $\begin{array}{c}18.01^{* * *} \\
(2.069)\end{array}$ & $\begin{array}{c}12.39^{* * *} \\
(3.035)\end{array}$ & $\begin{array}{c}2.057^{* * *} \\
(0.497)\end{array}$ \\
\hline HGS3 $*$ Quiz, $\gamma_{4}$ & $\begin{array}{c}3.584^{* * *} \\
(0.436)\end{array}$ & $\begin{array}{c}2.207^{* *} \\
(1.097)\end{array}$ & $\begin{array}{c}19.14^{* * *} \\
(1.565)\end{array}$ & $\begin{array}{c}10.01^{* * *} \\
(3.720)\end{array}$ & $\begin{array}{c}1.884^{* * *} \\
(0.588)\end{array}$ \\
\hline HGS3 $*$ Combin, $\gamma_{5}$ & $\begin{array}{c}3.692^{* * *} \\
(0.469)\end{array}$ & $\begin{array}{l}1.980^{*} \\
(1.033)\end{array}$ & $\begin{array}{c}16.99^{* * *} \\
(1.829)\end{array}$ & $\begin{array}{c}11.32^{* * *} \\
(3.192)\end{array}$ & $\begin{array}{c}1.857^{* * *} \\
(0.478)\end{array}$ \\
\hline Constant, $\alpha_{0}$ & $\begin{array}{c}2.813^{* * *} \\
(0.399)\end{array}$ & $\begin{array}{c}4.916^{* * *} \\
(0.906)\end{array}$ & $\begin{array}{c}19.23^{* * *} \\
(2.063)\end{array}$ & $\begin{array}{c}21.50^{* * *} \\
(1.942)\end{array}$ & $\begin{array}{c}3.989^{* * *} \\
(0.990)\end{array}$ \\
\hline Obs (Clusters) & $3168(144)$ & $792(36)$ & $3168(144)$ & $792(36)$ & $396(18)$ \\
\hline
\end{tabular}

Robust standard errors in parentheses, ${ }^{* * *} \mathrm{p}<0.01,{ }^{* *} \mathrm{p}<0.05,{ }^{*} \mathrm{p}<0.1$

Table 3: Regressions of Individual contributions and earnings as well as Minimum Contribution by group. 


\begin{tabular}{|c|c|c|c|c|}
\hline & \multicolumn{2}{|c|}{ " Original Low Group Members } & \multicolumn{2}{|c|}{ " Original High Group Members } \\
\hline & Before Own Move & After Own Move & Before Stage 3 & Stage 3 \\
\hline & \multicolumn{2}{|c|}{1 Period } & \multicolumn{2}{|c|}{1 Period } \\
\hline Baseline & 1.25 & 3.29 & 3.75 & 3.00 \\
\hline Quota & 0.77 & 4.35 & 4.00 & 4.13 \\
\hline Quiz & 1.21 & 4.89 & 6.00 & 6.25 \\
\hline \multirow[t]{2}{*}{ Combined } & 1.47 & 4.22 & 5.17 & 4.83 \\
\hline & \multicolumn{2}{|c|}{3 Periods } & \multicolumn{2}{|c|}{3 Periods } \\
\hline Baseline & 1.32 & 2.34 & 3.71 & 2.29 \\
\hline Quota & 0.99 & 4.49 & 4.08 & 4.04 \\
\hline Quiz & 1.31 & 4.60 & 6.08 & 5.71 \\
\hline Combined & 1.38 & 4.00 & 4.97 & 4.64 \\
\hline
\end{tabular}

Table 4: Summary statistics of contributions before and after group transitions.

the three treatment mechanisms. While we will not present all of the relevant test statistics, in every regression, the coefficients for the High group / Stage 3 interactions with the three treatments are statistically indistinguishable across treatments. That is, the beneficial effects of the three mechanisms are identical for contributions, earnings and minima. This indicates that either a quiz or quota restriction works equally well, and that combining the two kinds of entry restrictions yields no discernible additional benefits.

Another way of understanding the behavioral effects of the group transition mechanisms can be found in Tables 4 and 5 . Table 4 shows some summary statistics for average contributions for one and three periods before and after transitioning groups for subjects originally in Low groups and average contributions in the one and three periods before and after group transitions are allowed for those originally in High groups. These statistics provide a finer grained measure regarding how the subjects respond to group transitions. For those originally in the Low groups, the average contribution prior to move is approximately 1 in all treatments but after group transition it is above 4 in the three treatments with entry restrictions but only 2-3 in the Baseline. So there is a larger increase after moving groups in the three treatments than in the Baseline. For those originally in the High groups we measure behavior before and after group transitions are allowed which means we are looking at the last one or three periods of stage 2 versus the first one or three periods of Stage 3 . The table shows that for the three treatments there is a small increase or negligible drop in the periods right after group transitions are allowed but there is a larger initial drop in the Baseline condition and this drop worsens over the first 3 periods while that worsening does not occur in the three treatments. So it appears that those initially in the High Group drop their contributions rapidly as soon as group transitions are allowed while in the other three treatments contributions are stable.

Of course there are other elements in the experiments that could be responsible for some of these contribution differences. For example, contributions are likely affected by subjects' individual characteristics as well as by the prior round group minimum in the High group into which an entrant moving. Table 5 reports regressions testing the robustness of the above findings on how transitions affect contributions, controlling for those additional factors. The 


\begin{tabular}{|c|c|c|c|c|}
\hline & \multicolumn{4}{|c|}{ Contributions After Move / Stage 3 Begins } \\
\hline & \multicolumn{2}{|c|}{ Original LG Members } & \multicolumn{2}{|c|}{ Original HG Members } \\
\hline & 1 Period & 3 Periods & 1 Period & 3 Periods \\
\hline \multirow{2}{*}{$\begin{array}{l}\text { Group Minimum from } \\
\text { High Group } t-1\end{array}$} & $0.698^{* * *}$ & $0.565^{* * *}$ & $0.795^{* * *}$ & 0.478 \\
\hline & $(0.0781)$ & $(0.066)$ & $(0.0732)$ & $(0.421)$ \\
\hline \multirow{2}{*}{$\begin{array}{c}\text { Avg of Own Contributions } \\
x \text { Periods Before Move }\end{array}$} & 0.0825 & $0.354^{* * *}$ & - & 0.173 \\
\hline & $(0.138)$ & $(0.116)$ & & $(0.425)$ \\
\hline \multirow[t]{2}{*}{ Quota } & 0.524 & $1.755^{* * *}$ & $0.926^{*}$ & $1.566^{* * *}$ \\
\hline & $(0.449)$ & $(0.364)$ & $(0.473)$ & $(0.491)$ \\
\hline \multirow[t]{2}{*}{ Quiz } & 0.844 & $1.787^{* * *}$ & $1.460^{* *}$ & $1.930^{* * *}$ \\
\hline & $(0.511)$ & $(0.376)$ & $(0.602)$ & $(0.488)$ \\
\hline \multirow{2}{*}{ Combined } & 0.379 & $1.311^{* * *}$ & 0.706 & $1.451^{* * *}$ \\
\hline & $(0.448)$ & $(0.354)$ & $(0.549)$ & $(0.515)$ \\
\hline \multirow[t]{2}{*}{ Constant } & $0.832^{* *}$ & -0.139 & 0.0171 & -0.142 \\
\hline & $(0.392)$ & $(0.293)$ & $(0.331)$ & $(0.441)$ \\
\hline Observations & 114 & 111 & 36 & 36 \\
\hline$R^{2}$ & 0.565 & 0.668 & 0.880 & 0.836 \\
\hline
\end{tabular}

Table 5: Regressions on behavior before and after group transitions.

regressions use as the dependent variable the average of the contributions a subject made for the one or three periods after a group transition (Low subjects) or after group transitions are allowed (High subjects). The independent variables are the subject's own contributions in the previous one or three periods, the minimum in the High Group from the prior period, as well as the treatment binary variables.

For those moving from Low groups into High groups (first two regressions), we see that the immediate response is the same across all treatments but then diverges quickly. When looking only a single period on either side of a move to a High Group, on average all movers increase their contributions as a function of the minimum from the target group in the prior period as well as an additional increase of around 0.8. There are no differences across treatments. By looking three periods on either side though we see substantial differences between treatments. In the Baseline condition, movers still base their contributions on the group minimum from the target group in round prior to their move but the constant is insignificant indicating that there is no increase beyond that. Now the three dummy variables for the treatments all become significant and relatively large indicating that in those treatments subjects increase contributions over and above what their counterparts do in the Baseline. This means that the increase in contributions in the Baseline condition shown in table 4 is explained by subjects from the Low groups making some attempt to match the minimum contribution in the group they are moving into but perhaps imperfectly. The three treatment variables also have positive and statistically significant coefficients, indicating that contributions by entrants in these treatments increase following entry beyond any increase in the Baseline, and that this effect is not simply the result of entrants simply 
matching the higher previous High group minimums in those treatments. Those subjects originally in the High groups (second group of regressions) in the three treatment conditions evidence an increase their contributions once Stage 3 begins relative to the response by their counterparts in the Baseline condition. Again the response one round in is only moderately different between the treatments and the Baseline but becomes more substantial after only a few more periods.

\section{Conclusion}

This study explores the effectiveness of different mechanisms for allowing small, efficientlycoordinated groups to grow successfully by incorporating new group members from lowerperforming groups. We investigated three different mechanisms similar to ones commonly used outside of the laboratory to restrict entry. One was a simple Quota mechanism limiting the number of entrants in a period. The second was a Quiz through which entrants had to demonstrate a minimum level of knowledge of the growing group's history and of the incentive structure in our "production" environment. Finally, the third was a combination of the Quota and Quiz mechanisms. The results of the experiment show that any of the three mechanisms work equally as well in allowing successful growth without a substantial breakdown in efficient coordination, while our baseline treatment with no such restrictions on group entry generated almost complete breakdown in efficiency.

One way of interpreting these results is based on the fact that they show quite strong consistency with the findings in Weber (2006), which shows that well coordinated groups are able to sustain efficient high coordination when they grow in a controlled manner. But the current results substantially extend the prior ones. First, these results demonstrate that, not only can the small group grow successfully, but that the exact mechanism used to limit the influx of new members may be less important than the presence of some mechanism. This is a surprising and important finding as our results indicate that the success of the High groups was not based solely on limiting the speed of growth by limiting the number of new entrants. While the High Groups in the Quiz treatment lagged the High Groups in the Baseline by a period or so, their growth rates were otherwise quite similar. Yet the High groups in the Quiz treatment substantially outperformed those in the Baseline. There was, however, a negligible difference between the success of the High groups in the Quiz and the other two restricted treatments even though the High groups in those other two treatments grew at slower rates. Moreover, in Weber (2006) entry involved incorporating entrants with no history of playing the game, while here we show that successful growth can be achieved even when entrants come from groups that have previously faced a total collapse in coordination.

One might well imagine other reasons that the Quiz mechanism should have performed better than the Quota mechanism, as anyone who passed the quiz was guaranteed to have a good understanding of how payoffs are generated and to also know the history of successful coordination in the High group that they were about to join. Not only might this knowledge make entrants more likely to understand the need to maintain efficient coordination, but it might also have convinced incumbents in the well-coordinated High group that newcomers would not degrade their high contributions. Such screening was found to be quite important in a public goods setting in Ahn, Isaac and Salmon (2008, 2009), in which high contributing 
groups were able to maintain their high degree of cooperation only when existing group members were given the ability to approve or reject incoming members. In our Quota treatment there was no ability to screen, only a limitation on the numbers allowed to enter, and yet this mechanism worked equally as well as the Quiz in this coordination game environment.

We find the above results surprising, and indicative of a substitutability between limited entry without information and information without limited entry. When only one person enters the efficient group at a time, everyone is sufficiently secure that the efficient coordination will not be harmed even if the incumbents do not know much about that person. Similarly, when multiple people enter but those people have demonstrated relevant knowledge, everyone is similarly sufficiently confident that growth will not harm the efficient coordination. But the combination of the two mechanisms yields no discernible additional benefit. This suggests that as long as there is some mechanism to ensure that everyone is sufficiently confident that entry will not overwhelm efficiency, growth will be successful. Given that the only difference in growth rates occurred in the first round in which moves were allowed, this suggests that fear of what will happen with entry in that first round is critical - once groups have learned that they can grow successfully, they subsequently do so.

Given the pure coordination aspect of this game, such a story about beliefs regarding the choices of others being affected primarily by the number of incoming group members may well explain why each of these mechanisms performed equivalently. Given the difference in strategic incentives between a coordination game such as the one we study here and a public goods game, this leads to an obvious and interesting next question of whether or not this property holds in that environment as well. Given that the public goods paradigm is the main other framework one might use to explain the dynamics of group production, this is certainly an interesting and important avenue for further study.

\section{References}

Ahn, T., R. M. Isaac, and T. C. Salmon (2008). Endogenous Group Formation. Journal of Public Economic Theory 10(1), 171-194.

Ahn, T., R. M. Isaac, and T. C. Salmon (2009). Coming and Going: Experiments on Endogenous Group Sizes for Excludable Public Goods. Journal of Public Economics 93(12), 336-351.

Borjas, G. J. (1994). The economics of immigration. Journal of Economic Literature 32(4), $1667-1717$.

Cable, D. M. and T. A. Judge (1996). Person-organization fit, job choice decisions, and organizational entry. Organizational Behavior and Human Decision Processes 67(3), 294311.

Card, D. (2005). Is the new immigration really so bad. Economic Journal 115(507), $300-323$. 
Chatman, J. A. and F. J. Flynn (2001). The influence of demographic composition on the emergence and consequences of cooperative norms in work teams. Academy of Management Journal 44(5), 956-974.

Fischbacher, U. (2007). z-Tree: Zurich Toolbox For Readymade Economic Experiments. Experimental Economics 10(2), 171-178.

Friedberg, R. M. (2001). The impact of mass migration on the israeli labor market. The Quarterly Journal of Economics 116(4), 1373-1408.

O' Reilly III, C. A., J. Chatman, and D. F. Caldwell (1991). People and organizational culture: A profile comparison approach to assessing person-organization fit. The Academy of Management Journal 34(3), 487-516.

Ortega, F. (2005). Immigration quotas skill upgrading. Journal of Public Economics 89(910), 1841-1863.

Rumbaut, R. G. (1997). Immigration and incorporation. Sociological Perspectives 40(3), $333-338$.

Ryan, A. M. and P. R. Sackett (1987). Pre-employment honesty testing: Fakability, reactions of test takers and company image. Journal of Business and Psychology 1(3), $248-256$.

Van Huyck, J. B., R. C. Battalio, and R. O. Beil (1990). Tacit coordination games, strategic uncertainty, and coordination failure. The American Economic Review 80(1), 234-248.

Warne, F. J. (1921). Assimilation and governmental regulation. The Annals of the American Academy of Political and Social Science 93, 181-185.

Weber, R. A. (2006). Manging growth to achieve efficient coordination in large groups. The American Economic Review 96(1), 114-126. 